\title{
Método para análise cinemática dos chutes de lactentes
}

\author{
Method for the kinematic analysis of kicking movement in infants \\ Método para el análisis cinemático de los pataleos de lactantes \\ Jocelene de Fátima Landgraf', Raquel de Paula Carvalho², Eloisa Tudella ${ }^{3}$
}

\begin{abstract}
RESUMO I Os objetivos deste estudo foram descrever o método utilizado para a análise cinemática dos movimentos de chutes em lactentes e testar sua viabilidade de uso, empregando o sistema Dvideow. Para adequação do método, quatro lactentes foram filmados, longitudinalmente, nas idades de um a seis meses na posição supina. O experimento teve a duração de dois minutos, subdivididos em duas condições: treinamento e observação. O sistema Dvideow 6.3 foi utilizado para análise das imagens. Foi verificado que o uso de quatro câmeras de vídeo é mais apropriado para garantir a visibilidade de dois marcadores, simultaneamente, permitindo a reconstrução tridimensional do movimento. Além disso, utilizamos 6 fios de prumo para calibrar o sistema e garantir uma precisão de $2 \mathrm{~mm}$. Portanto, concluímos que a utilização do sistema Dvideow para realizar a análise cinemática dos chutes de lactentes mostrou-se adequada e viável, uma vez que esse sistema é acessível, de baixo custo e de fácil utilização para os pesquisadores em geral.

Descritores I lactente; extremidade inferior; desenvolvimento infantil; biomecânica.
\end{abstract}

ABSTRACT I The aims of this study were to describe the method used for the kinematic analysis of kicking movements in infants and test its feasibility of use, using the Dvideow system. To adjust the method, four infants were longitudinally videotaped at ages from one to six months in the supine position. The experiment lasted two minutes, divided into two conditions: training and observation. The Dvideow system 6.3 for image analysis was used. It was verified that the use of four video cameras was more appropriate for the visibility of two markers simultaneously, allowing 3D reconstruction of motion. Moreover, 6 plumb lines were used to calibrate the system and ensure an accuracy of $2 \mathrm{~mm}$. It was concluded that the use of the Dvideow system to perform kinematic analysis of the kicking movement in infants proved to be appropriate and feasible, since this system is accessible, of low cost and easy to be used for researchers in general.

Keywords I infant; lower extremity; child development; biomechanics.

RESUMEN I Los objetivos de este estudio fueron describir el método utilizado para el análisis cinemático de los movimientos de pataleo en lactantes y probar su factibilidad de uso, empleando el sistema Dvideow. Para la adecuación del método, cuatro lactantes fueron filmados, longitudinalmente, en las edades de uno a seis meses en la posición supina. El experimento tiene una duración de dos minutos, subdivididos en dos condiciones: entrenamiento y observación. El sistema Dvideow 6.3 fue utilizado para el análisis de las imágenes. Fue verificado que el uso de cuatro cámaras de video es más apropiado para garantizar la visibilidad de dos marcadores simultáneos, permitiendo la reconstrucción tridimensional del movimiento. Además, utilizamos seis alambres de plomo para calibrar el sistema y garantizar una precisión de $2 \mathrm{~mm}$. Por lo tanto, concluimos que la utilización del sistema Dvideow para realizar el análisis cinemático de los pataleos de lactantes se mostró adecuada y viable, ya que éste sistema es accesible, de bajo costo y de fácil utilización para los investigadores en general.

Palabras clave I lactantes; miembros inferiores; desarrollo; cinemática.

Estudo desenvolvido no Laboratório de Pesquisa e Análise do Movimento do Departamento de Fisioterapia da Universidade Federal de São Carlos (UFSCar) - São Carlos (SP), Brasil.

'Doutora em Fisioterapia; Professora Adjunta da Universidade Federal do Rio de Janeiro (UFRJ) - Rio de Janeiro (RJ), Brasil.

${ }^{2}$ Doutora em Fisioterapia; Professora Adjunta da Universidade Federal de São Paulo (UNIFESP) - Santos (SP), Brasil.

${ }^{3}$ Doutora em Psicologia; Professora Adjunta da UFSCar - São Carlos (SP), Brasil.

Endereço para correspondência: Jocelene de Fátima Landgraf - Departamento de Clínica Médica - Rua Prof. Rodolpho Paulo Rocco, s/n, Hospital Universitário Clementino Fraga Filho, 80 andar, ala E, sala 3 - Illha do Fundão - CEP: 21910-590 - Rio de Janeiro (RJ), Brasil - E-mail: jolandgraf@gmail.com

Apresentação: fev. 2012 - Aceito para publicação: fev. 2013 - Fonte de financiamento: Fundação de Amparo à Pesquisa do Estado de São Paulo - Conflito de interesse: nada a declarar - Parecer de aprovação do Comitê de Ética nº 044/2005. 


\section{INTRODUÇÃO}

Nas últimas décadas, para estudar o movimento de lactentes com desenvolvimento típico ou atípico, os pesquisadores têm utilizado a análise cinemática. Analisar cinematicamente o movimento consiste em obter coordenadas espaciais referentes à localização de pontos anatômicos definidos no corpo, durante a realização de uma ação motora. Utilizando essas coordenadas, é possível obter variáveis quantitativas que descrevem o movimento dos segmentos corporais. Especialmente na área de desenvolvimento motor, observa-se avanços na compreensão da aquisição e aprimoramento de diversas habilidades motoras em lactentes, sendo a análise cinemática o método mais empregado em estudos sobre o alcance manual ${ }^{1-4}$, os chutes espontâneos ${ }^{5,6}$ e a marcha ${ }^{7-9}$.

Os chutes espontâneos são exemplos dos comportamentos mais precoces observados em lactentes ${ }^{10}$ e acredita-se que desempenham importante papel no desenvolvimento da locomoção bípede ${ }^{11}$. Por meio de análise cinemática, foi demonstrado que os chutes espontâneos apresentam estrutura espacial e temporal semelhantes à locomoção humana madura ${ }^{12}$. Alguns autores verificaram a influência do móbile nos chutes espontâneos, demonstrando que lactentes aos quatro meses de idade aprenderam a tocar um painel com os pés para ativar o móbile ${ }^{10}$. Outros autores investigaram o efeito do peso unilateral em lactentes nas idades de 6,12,18 e 26 semanas, o que resultou em mudanças no comportamento de chute, nas idades de $6 \mathrm{e}$ 12 semanas, na frequência de chutes, na amplitude de movimento e no pico de velocidade de ambas as pernas ${ }^{13}$; e investigaram a contribuição do trato corticoespinhal na regulação e coordenação intermembro e na organização espaço-temporal dos chutes de lactentes com e sem leucomalácia periventricular submetidos ao acréscimo de peso unilateral, encontrando algumas diferenças, que não puderam ser atribuídas às influências corticoespinhais ${ }^{14}$. Para tanto, sistemas de análise como OPTOTRAK 3020 motion analysis system sensors (Northern Digital Inc., Waterloo, Ont., Canadá) e VIDIPLUS (Vrije Universiteit, Amsterdã, Holanda) têm sido utilizados no estudo dos chutes de lactentes. Dessa forma, ressalta-se a importância dos estudos que exploram esse comportamento específico, ampliando os conhecimentos da área de desenvolvimento e comportamento motor. Entretanto, a metodologia específica para a realização desses estudos pode não ser acessível a toda comunidade científica devido ao alto custo dos sistemas disponíveis no mercado.

No Brasil, utilizamos o sistema Dvideow para o estudo do movimento de lactentes, o qual tem sido aplicado nos estudos do alcance manua $1^{15}$ e da coordenação visocefálica ${ }^{16}$. Além disso, pela facilidade do uso, esse sistema pode ser empregado para o estudo de inúmeros comportamentos motores.

O sistema Dvideow (Digital Video for Biomechanics for Windows 32 bits) foi desenvolvido pelas equipes do Laboratório de Instrumentação Biomecânica (Faculdade de Educação Física) e do Instituto de Computação da Universidade Estadual de Campinas (UNICAMP), e consiste fundamentalmente em equipamentos não dedicados e um programa de computador ${ }^{17,18}$. Algumas vantagens na utilização desse sistema são o fato de que o custo do sistema é menor em comparação aos demais. Além disso, como ocorre com outros equipamentos, não é necessário o uso de cabos, e os marcadores podem se adequar aos estudos com lactentes. Segundo os autores do sistema Dvideow, é possível que características próprias dos marcadores posicionados nos pontos anatômicos do corpo possam ser utilizadas como padrão a ser identificado na sequência de imagens, permitindo o rastreamento automático desses pontos durante todo o movimento. Dessa forma, a reconstrução tridimensional do movimento pode ser obtida de forma rápida e precisa, otimizando o tempo de análise.

Apesar do sistema ter sido utilizado na reconstrução tridimensional do movimento de adultos e bebês, o mesmo ainda não havia sido empregado para a reconstrução dos chutes espontâneos. Sendo assim, fez-se necessário um estudo metodológico para verificar aspectos como o posicionamento e o número de câmeras, definição do sistema de calibração, colocação dos marcadores, reconstrução dos movimentos e definição das variáveis. Portanto, os objetivos deste trabalho foram descrever o método utilizado para a análise cinemática dos movimentos de chutes em lactentes e testar sua viabilidade de uso, empregando o sistema Dvideow.

\section{METODOLOGIA}

O método será descrito de acordo com as etapas necessárias para a reconstrução tridimensional do movimento: 1) montagem do sistema de calibração; 2) posicionamento e número de câmeras; 3) procedimentos de teste.

\section{Sistema de calibração}

Para que a reconstrução tridimensional do movimento seja realizada, é necessário que o movimento aconteça 
dentro de um volume calibrado com medidas conhecidas. Para tanto, foram testados dois sistemas de calibração. O sistema 1 foi composto por 4 fios de aço, de 2,30 m de comprimento, formando um cubo no centro da sala de avaliação de dimensões $0,48 \times 0,32 \times 0,35 \mathrm{~m}^{3}$. $\mathrm{Na}$ extremidade inferior de cada fio, foi fixado um cone de chumbo de $50 \mathrm{~g}$. Ao longo dos fios foram fixados 10 marcadores ( $1 \mathrm{~cm}$ de diâmetro), a uma distância de $10 \mathrm{~cm}$ entre si, conforme mostra a Figura 1A. Para o sistema 2 , foi construído um volume composto por seis fios de aço, de 2,30 m de comprimento, dispostos de modo a formar um retângulo no centro da sala com volume de $0,64 \times 0,84 \times 0,35 \mathrm{~m}^{3}$. $\mathrm{Na}$ extremidade inferior de cada fio, foi fixado um cone de chumbo de $400 \mathrm{~g}$. Ao longo dos fios foram fixados 25 marcadores ( $0,5 \mathrm{~cm}$ de diâmetro), a uma distância de $5 \mathrm{~cm}$ entre si, conforme Figura 1B. As coordenadas XYZ de ambos os sistemas foram aferidas utilizando-se um teodolito mecânico e uma trena de $3 \mathrm{~m}$ com graduação em milímetros. As coordenadas $\mathrm{X}$ e $\mathrm{Y}$ eram coordenadas planas, e a coordenada $\mathrm{Z}$ foi tomada como sendo a diferença de altura entre os marcadores e o ponto de origem do sistema de referências. Foi realizado um teste de acurácia para verificar a margem de erro de ambos os sistemas de calibração. Para

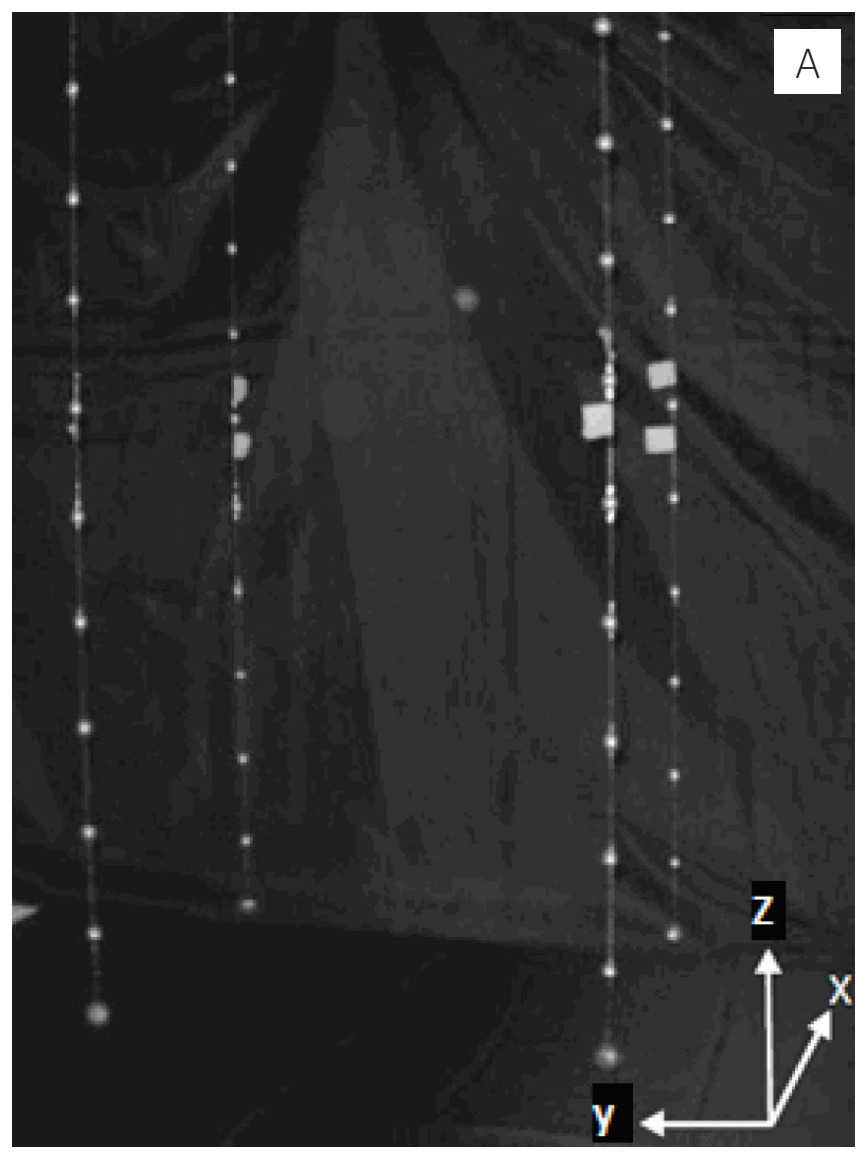

tanto, foi utilizada uma haste com dois marcadores fixados nas extremidades superiores, com uma distância de $30 \mathrm{~cm}$ entre si, que foi filmada enquanto o examinador a movimentava em diferentes direções dentro do volume, em ambos os sistemas de calibração, durante um minuto. Posteriormente, essas imagens foram reconstruídas; a distância entre os marcadores da haste e a acurácia foram calculadas pela seguinte fórmula: $\mathrm{A}^{2}=\mathrm{b}^{2}+\mathrm{p}^{2}$, onde $\mathrm{b}$ é bias das medidas, dado pelo desvio entre o valor médio do conjunto de medidas e o valor esperado, e $\mathrm{p}$ é a medida de dispersão de um conjunto de dados em relação ao valor médio, dado pelo desvio padrão das medidas experimentais ${ }^{19}$.

\section{Posicionamento e número de câmeras}

Considerando que o sistema Dvideow permite a utilização de até seis câmeras de vídeo e que os segmentos de interesse eram os membros inferiores dos lactentes, verificou-se a necessidade de dividir a análise em duas, uma para cada membro inferior. Para registro dos dados, duas conformações de posicionamento e número de câmeras eram possíveis. Para a conformação 1 , foram utilizadas três câmeras de vídeo digitais JVC (modelo GY

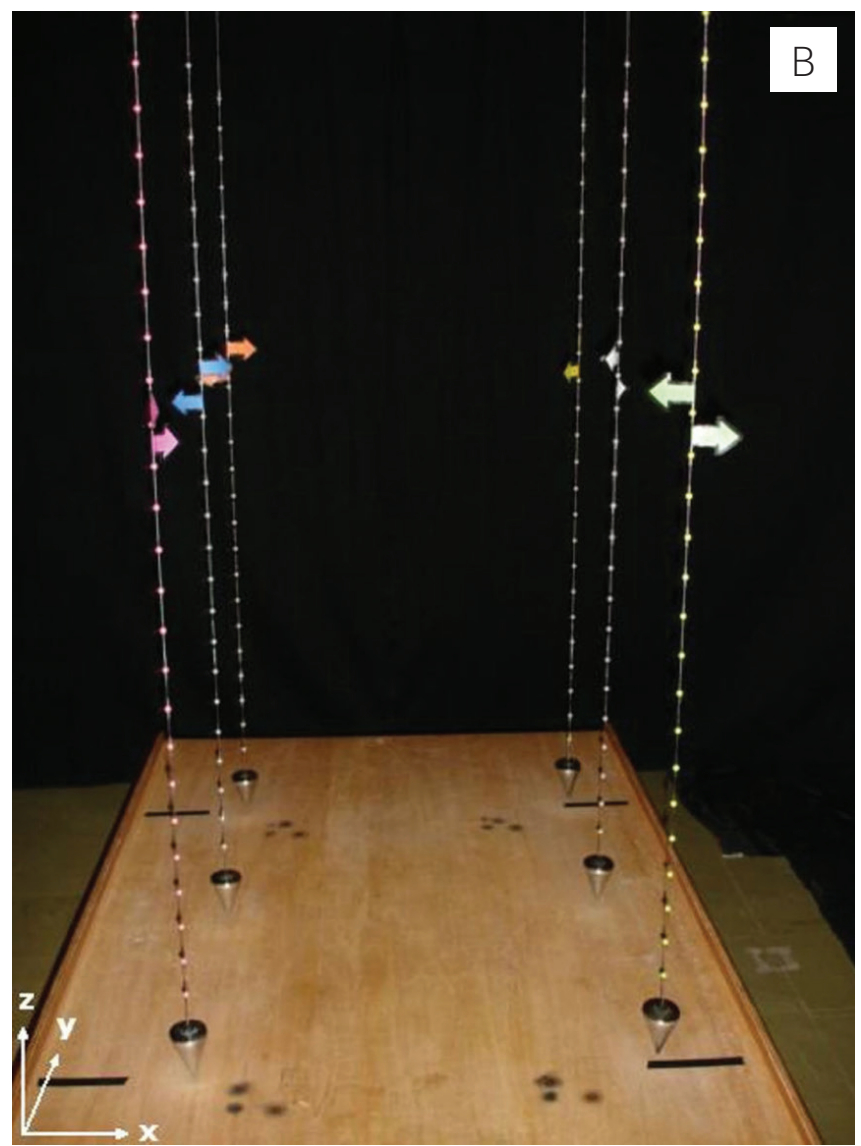

Figura 1. Calibração. (A) Sistema 1, com quatro fios; (B) Sistema 2, com seis fios 
DV-300), posicionadas em tripés. Uma câmera foi posicionada posteriormente à mesa de exame a uma altura de $2 \mathrm{~m}$ do solo, enquanto as outras duas foram posicionadas anterolateralmente ao tablado, formando um ângulo de $60^{\circ}$ entre si, com altura de $1,2 \mathrm{~m}$ em relação ao solo. Para análise do movimento realizado com o membro inferior direito, foram analisadas as imagens das câmeras da direita e posterossuperior, e para análise dos chutes com membro inferior esquerdo foram analisadas as imagens das câmeras da esquerda e posterossuperior.

Para a conformação 2, foram utilizadas quatro câmeras de vídeo digitais, sendo três JVC (modelo GY DV-300) e uma Sony (DCR-TRV30), acopladas a tripés. Duas câmeras foram posicionadas de cada lado do tablado, formando um ângulo de $120^{\circ}$ entre si, sendo as câmeras da direita usadas para registrar as imagens do membro inferior direito, e as da esquerda, para o membro inferior esquerdo. A altura das câmeras em relação ao solo foi de aproximadamente 1,4 m. As duas conformações foram testadas de acordo com a quantidade de oclusão dos marcadores posicionados nos membros inferiores durantes os testes. Tais conformações podem ser observadas na Figura 2.

Dois iluminadores (com lâmpada de $500 \mathrm{~W}$ ) com difusores foram direcionados para a parede a fim de que os marcadores refletissem adequadamente e não incomodassem a visão dos lactentes.

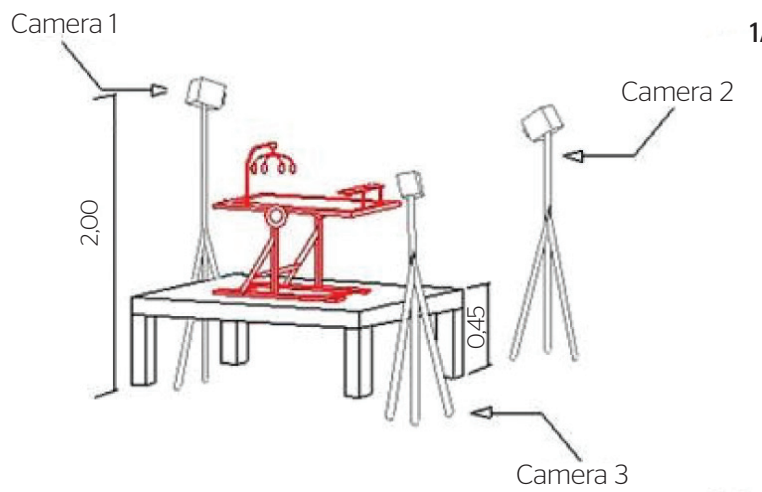

$1 \mathrm{~A}$

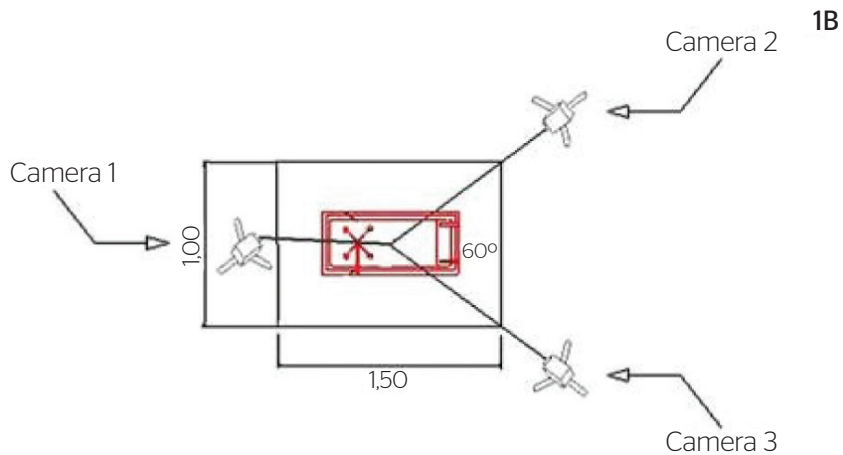

Após a definição do sistema de calibração e do posicionamento e número das câmeras, foram realizados os procedimentos de teste com os bebês para analisarmos a viabilidade de tal metodologia para a reconstrução dos chutes espontâneos.

Antes de cada coleta de dados, foi realizada a calibração do sistema, que consistiu em programar a câmera para controle manual para ajustar o balanço de branco, foco e velocidade de abertura do obturador, de acordo com a iluminação utilizada e a precisão desejada. O enquadramento da imagem foi realizado para que os fios de prumo fossem filmados por período de um segundo. A seguir, foram retirados os fios de prumo e posicionada a mesa de exame infantil, e as câmeras permaneceram ligadas até a finalização da avaliação com o objetivo de que os ajustes feitos na câmera não se alterassem, garantindo a fidedignidade das medidas aferidas.

\section{Procedimentos de teste}

Para analisar o método para o estudo dos chutes, foi necessário testar uma amostra de quatro lactentes sadios, sendo dois do sexo masculino e dois do feminino, nascidos a termo $(38,75 \pm 0,5$ semanas de idade gestacional), com índice de Apgar igual ou superior a 8 no $1^{\circ} \mathrm{mi}-$ nuto $(8,25 \pm 0,5)$ e 9 no $5^{\circ}$ minuto $(9,25 \pm 0,5)$ e com
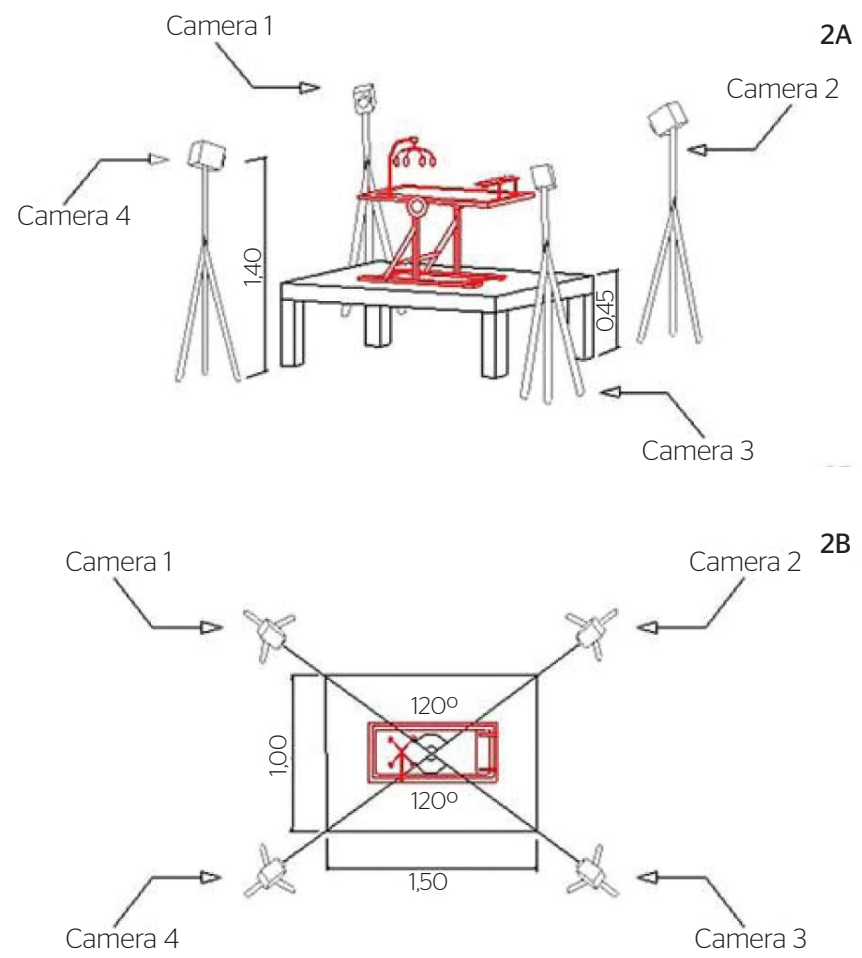

Figura 2. Arranjo experimental: 1A e 2A: perspectiva esquemática dos sistemas 1 e 2, respectivamente; $1 \mathrm{~B}$ e 2B: planta esquemática dos sistemas 1 e 2 , respectivamente 
peso adequado à idade gestacional $(3.331 \pm 416,62 \mathrm{~g})$. Os sujeitos foram selecionados por meio da consulta de prontuários em uma Unidade Básica de Saúde. Durante a avaliação, o lactente deveria estar em estado de alerta inativo ou ativo (graus 3 e 4$)^{20}$. A avaliação foi realizada no intervalo entre as mamadas, para que a fome ou a saciedade não interferisse no comportamento do lactente.

O presente estudo foi aprovado pelo Comitê de Ética em Pesquisa com Seres Humanos da Universidade Federal de São Carlos (UFSCar), e os pais dos lactentes assinaram o termo de consentimento livre e esclarecido. $\mathrm{O}$ estudo foi realizado no Laboratório de Pesquisa e Análise do Movimento (LaPAM) da UFSCar.

Os quatros lactentes foram filmados uma vez ao mês, do primeiro ao sexto mês de idade. Essas avaliações foram realizadas nas datas de aniversário, considerando um intervalo de sete dias antes ou após a data.

No laboratório, o lactente era despido pela mãe e o examinador coletava os dados antropométricos de peso, altura e comprimentos dos membros inferiores dos lactentes. Em seguida, foram fixados marcadores bilateralmente no tronco (intersecção da linha média e última costela) e nos membros inferiores, nas seguintes referências anatômicas: espinha ilíaca anterossuperior, trocânter do fêmur, epicôndilo lateral do fêmur, maléolo lateral e base do quinto metatarso ${ }^{21}$.

Os lactentes foram posicionados em supino na mesa de exame infantil ${ }^{22}$. $\mathrm{Na}$ extremidade inferior da mesa, foi fixado um painel $(0,30 \times 0,10 \mathrm{~m})$, que ao ser elevado acionava um móbile (Figura 3) com estímulo visual e auditivo, como reforço para incentivar os movimentos de chute ao painel. As medidas de comprimento do membro inferior foram usadas para a definição

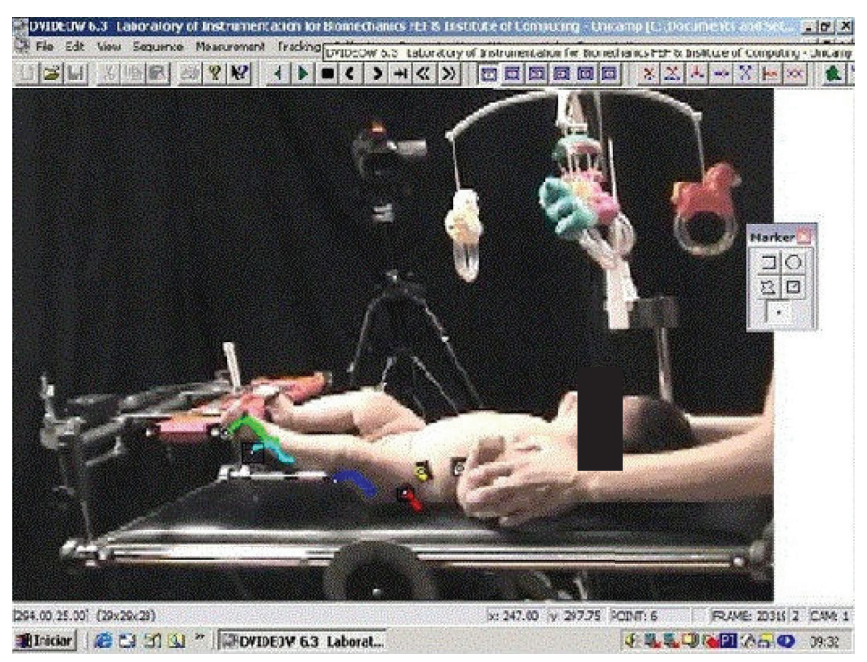

Figura 3. Interface do sistema Dvideow 6.3 e rastreamento dos marcadores do posicionamento da altura do painel fixado à mesa: $\left[\text { (comprimento da perna) } \mathrm{x} \text { sen }\left(30^{\circ}\right)\right]^{10}$. Para manter as condições de segurança do lactente e evitar o deslocamento do seu corpo durante os movimentos de chute, uma faixa e/ou um auxiliar de pesquisa, posicionado posteriormente à cadeira, oferecia estabilidade ao lactente, mantendo-o pela cintura escapular.

O experimento teve a duração aproximada de dois minutos subdivididos em duas condições: 1) Treinamento (T) - segurando os tornozelos dos lactentes, o examinador colocava o pé direito, em seguida o pé esquerdo e, finalmente, os dois pés simultaneamente, por três vezes consecutivas no painel a fim de elevá-lo para acionar o móbile; nesse momento, o móbile girava e emitia uma música infantil com o objetivo de estimular o movimento ensinado; 2) Observação $(\mathrm{O})$ - lactente posicionado em supino por um minuto na mesa de exame, podendo, durante os chutes, elevar o painel com os pés e acionar o móbile.

As imagens foram repassadas para o computador por uma placa de captura de imagens, utilizando-se o software Studio 9.1, em formato de arquivos AVI. Foram considerados movimentos de chute toda vez que o lactente realizava movimento de extensão de quadril e joelho de um ou ambos os membros inferiores em direção ao painel. $\mathrm{O}$ início do movimento de chute foi estabelecido como sendo o quadro que mostrou o primeiro deslocamento dos marcadores posicionados no trocânter do fêmur e no maléolo lateral.

As imagens foram processadas no sistema Dvideow 6.3, que fornece, como resultado, as coordenadas $\mathrm{X}, \mathrm{Y}$ e $\mathrm{Z}$ de cada marcador em cada quadro do movimento analisado (Figura 3).

A partir disso, foi utilizado o programa Matlab 6.1, com o objetivo de filtrar e processar esses dados. Um filtro do tipo Butterworth digital de $4^{\mathrm{a}}$ ordem, com cut off de $6 \mathrm{~Hz}$ foi aplicado nas coordenadas dos movimentos.

O cálculo da variação angular de quadril, joelho e tornozelo, definido como os ângulos de quadril, joelho e tornozelo desde o início até o final dos chutes foi verificado.

\section{RESULTADOS E DISCUSSÃO}

Nos testes de acurácia de ambos os sistemas de calibração, obteve-se para o sistema 1 uma margem de erro de $7 \mathrm{~mm}$, enquanto, para o sistema 2, o erro foi de $2 \mathrm{~mm}$. Esse resultado justifica-se porque o sistema 2 fornecia um maior número de marcadores com medidas 
conhecidas no volume de calibração, o que garantiu maior precisão na reconstrução do movimento. Outros estudos demonstraram acurácia de $1 \mathrm{~mm}^{10}$ e menor que $3 \mathrm{~mm}^{21}$, o que demonstra que a acurácia do nosso sistema está compatível com a de trabalhos realizados por outros autores, utilizando outros sistemas. Ambos os cones de chumbo utilizados nos dois sistemas, apesar de terem pesos diferentes ( $50 \mathrm{~g}$ no sistema 1 e $400 \mathrm{~g}$ no sistema 2), foram eficientes para atender o propósito de manter os fios estirados e estabilizados durante a calibração do sistema.

De acordo com os dois outros estudos realizados em nosso laboratório utilizando o sistema Dvideow, verificou-se que a utilização de três câmeras é adequada para a análise cinemática do alcance manua $1^{15}$ e da coordenação viso-cefálica ${ }^{16}$, mas não para os estudos dos chutes de lactentes. Na configuração 1 , a câmera 1 , posicionada posterossuperiormente à mesa de avaliação, não permitia que todos os marcadores fossem visualizados, especialmente quando o lactente realizava a extensão de quadril com rotação lateral, prejudicando o rastreamento automático dos pontos durante a análise pelo Dvideow. Dessa forma, para o estudo da cinemática dos chutes, quatro câmeras foram necessárias; sendo dispostas duas de cada lado da mesa de exame infantil, formando um ângulo de $120^{\circ}$ entre si, os marcadores do tronco e de cada membro inferior do lactente eram vistos por duas câmeras simultaneamente, requisito para reconstrução tridimensional.

A idade dos lactentes também foi importante na observação dos chutes, uma vez que de recém-nascido a quatro meses há grande frequência de chutes. No quinto e no sexto mês, foram observados poucos comportamentos de chute, pois os lactentes demonstraram maior interesse em levar os pés à boca e em rolar. Na literatura, verificamos que vários estudos foram realizados no intuito de observar os chutes nos primeiros meses ${ }^{12,23,24} \mathrm{e}$ uma quantidade menor foi realizada para verificar esse comportamento após o quinto mês ${ }^{25}$.

Os marcadores de $0,5 \mathrm{~cm}$ de diâmetro refletiram adequadamente para visualização no sistema Dvideow, desde que utilizando iluminadores na sala. A posição de marcadores foi adequada e permitiu verificar a variação angular de quadril, joelho e tornozelo durante os chutes. Essa variação angular está ilustrada na Figura 4 para lactentes com 1,2, 3 e 4 meses de idade.

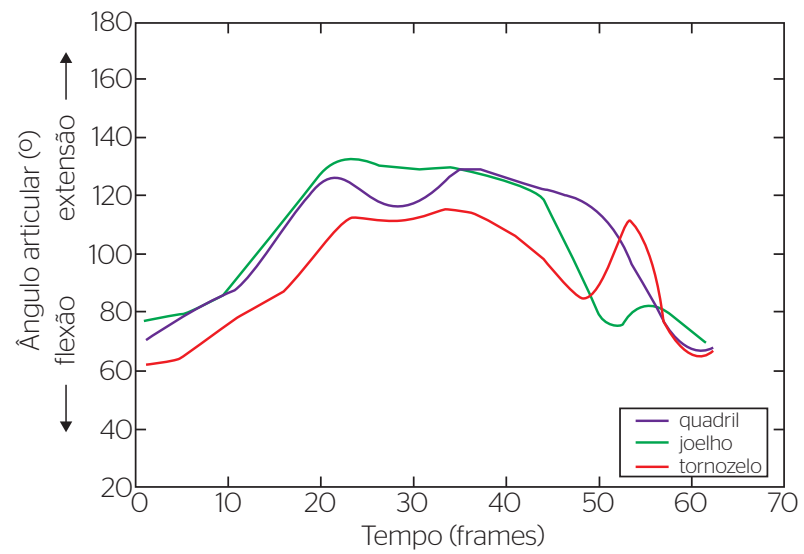

A
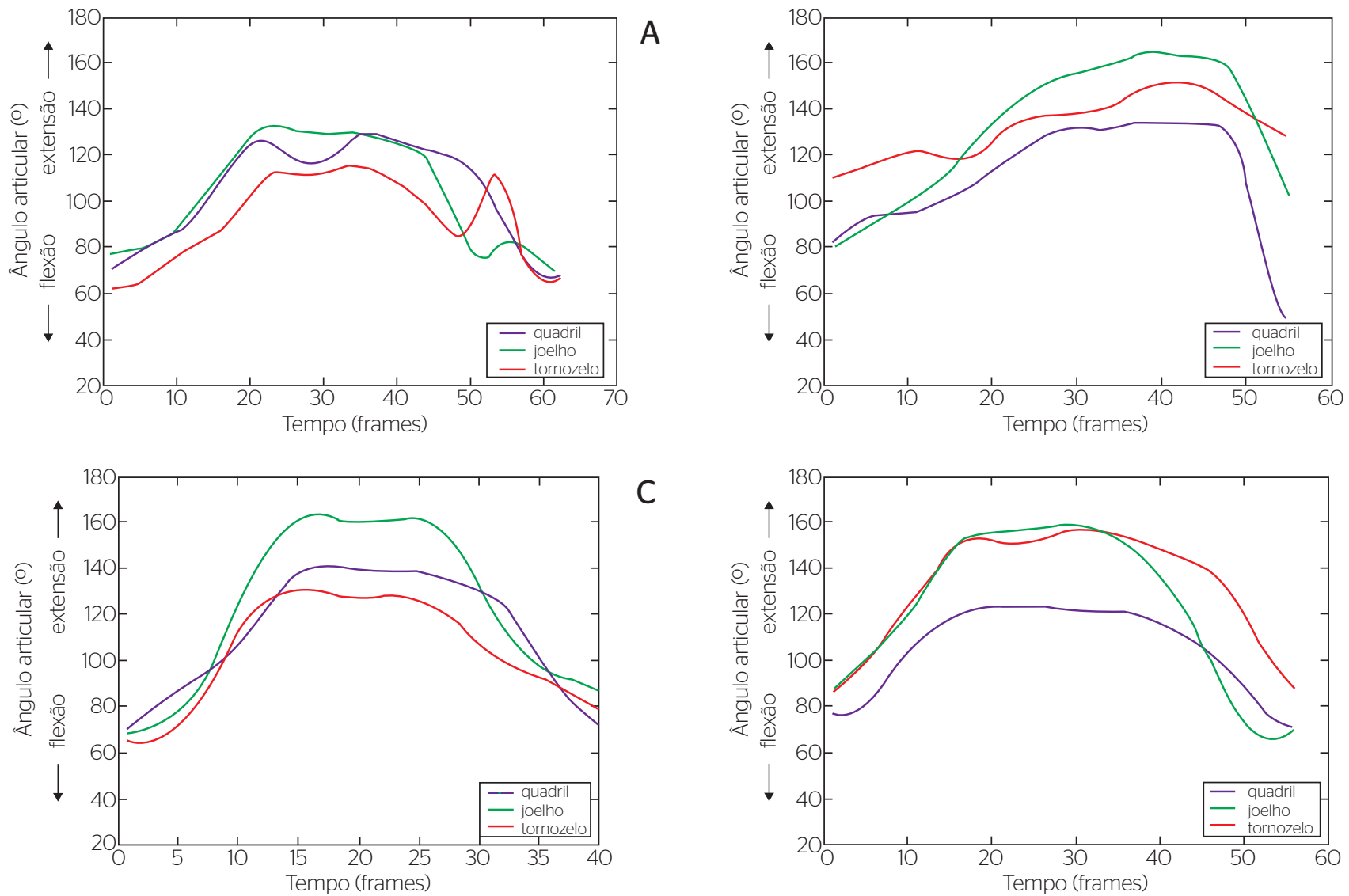
Sobre a fixação do lactente à mesa de exame infantil, verificamos que utilizar a faixa na altura do tórax foi uma limitação da pesquisa, pois quando o lactente tocava o painel com os pés, ele conseguia se impulsionar para cima, estando com a faixa, e o posicionamento ficava comprometido. Além disso, a faixa na altura do tórax do lactente dificultou a visualização do marcador posicionado no tronco. Para resolver esse problema, um examinador estabilizou o lactente pela cintura escapular.

Também verificamos que diversos estudos foram realizados para investigar os chutes de lactentes e a influência que fatores intrínsecos ou extrínsecos exercem sobre eles. Com o aparato utilizado neste estudo e o sistema Dvideow, pudemos verificar que isso também é possível. Podemos verificar o comportamento de chutes com o lactente chutando livremente por tempo predeterminado; verificar como ele reage com a colocação do painel e do móbile musical; verificar o efeito que o acréscimo de peso uni ou bilateral exerce; realizar os estudos com lactentes de risco ou lesões neurológicas, dentre outras possibilidades. Em cada estudo, somente precisa ficar claro quais grupos (diferentes idades, lactentes com síndromes ou lesões encefálicas, dentre outros) e quais variáveis (frequência de chutes, duração, preferência podal, velocidade, dentre outras) são analisadas. Um diferencial do nosso estudo em relação a outros realizados com os chutes de lactentes é que podemos acrescentar a variável índice de retidão, pois utilizamos um painel (alvo) para ser atingido pelo pé do lactente. $O$ índice de retidão compara a trajetória realizada pelo pé em relação à menor distância que o pé poderia ter percorrido. Essa variável foi utilizada anteriormente em estudos sobre o alcance manual ${ }^{3}$ e não observada em estudos que analisaram os chutes.

\section{CONCLUSÃO}

Todo o método empregado foi adequado para a análise cinemática dos chutes de lactentes. $\mathrm{O}$ posicionamento dos marcadores e das câmeras e as variáveis estudadas forneceram subsídios para entender os chutes. A utilização do sistema Dvideow para realizar a análise cinemática dos chutes de lactentes mostrou-se adequada e viável, uma vez que esse sistema é acessível, de baixo custo e de fácil utilização para os pesquisadores em geral. Além disso, como requer apenas que marcadores sejam fixados em pontos específicos, permite que os lactentes tenham liberdade de movimentação.

\section{REFERÊNCIAS}

1. Bakker H, de Graff-Peters VB, van Eykern LA, Otten B, Hadders-Algra $M$. Development of proximal arm muscle control during reaching in young infants: from variation to selection. Infant Behav Dev. 2010;33(1):30-8.

2. Toledo AM, Tudella E. The development of reaching behavior in lowrisk preterm infants. Infant Behav Dev. 2008;31(3):398-407.

3. Carvalho RP, Tudella E, Caljouw SR, Savelsbergh GJ. Early control of reaching: effects of experience and body orientation. Infant Behav Dev. 2008;31(1):23-33.

4. Lee HM, Bhat A, Scholz JP, Galloway JC. Toy-oriented changes during early arm movements IV: shoulder-elbow coordination. Infant Behav Dev. 2008;31(3):447-69.

5. Domellöf E, Rönnqvist L, Hopkins B. Functional asymmetries in the stepping response of the human newborn: a kinematic approach. Exp Brain Res. 2007:177(3):324-35.

6. Fetters L, Chen YP, Jonsdottir J, Tronick EZ. Kicking coordination captures differences between full-term and premature infants with white matter disorder. Hum Mov Sci. 2004;22(6):729-48.

7. Wu J, Looper J, Ulrich DA, Angulo-Barroso RM. Effects of various treadmill interventions on the development of joint kinematics in infants with Down syndrome. Phys Ther. 2010;90(9):1265-76.

8. Karol LA, Jeans K, ElHawary R. Gait analysis after initial nonoperative treatment for clubfeet: intermediate term followup at age 5. Clin Orthop Relat Res. 2009;467(5):1206-13.

9. Jeng SF, Lau TW, Hsieh WS, Luo HJ, Chen PS, Lin KH, Shieh JY. Development of walking in preterm and term infants: age of onset, qualitative features and sensitivity to resonance. Gait Posture. 2008:27(2):340-6

10. Chen YP, Fetters L, Holt KG, Saltzman E. Making the mobile move: constraining task and environment. Inf Behav Dev. 2002;25(2):195-220.

11. Thelen E. Developmental origins of motor coordination leg movements in human infants. Dev Psychobiol. 1985;18(1):1-22.

12. Thelen E, Bradshaw G, Ward JA. Spontaneous kicking in month-old infants: manifestation of a human central locomotor program. Behav Neural Biol. 1981:32:45-53.

13. Vaal J, van Soest AJK, Hopkins B. Spontaneous kicking in infants: age-related effects of unilateral weighting. Dev Psychobiol. 2000;36(2):111-122

14. Vaal J, van Soest AJK, Hopkins B, Sie LTL. Spontaneous leg movements in infants with and without periventricular leukomalacia: effects of unilateral weighting. Behav Brain Res. 2002:129(1-2):83-92.

15. Carvalho RP, Tudella E, Barros RML. Utilização do sistema Dvideow na análise cinematic do alcance manual de lactentes. Rev Bras Fisioter. 2005;9(1):1-7.

16. Lima CD, Carvalho RP, Barros RML, TudellaE. Dois métodos diferentes para análise cinematica dos movimentos de cabeça durante a coordenação viso-cefálica de lactentes. Rev Bras Fisioter. 2008;12(5):425-31.

17. Barros RML, Brenzikofer R, Leite NJ, Figueroa PJ. Desenvolvimento e avaliação de um sistema para análise cinemática tridimensional de movimentos humanos. Rev Bras Eng Biomed. 1999;15(1-2):79-86.

18. Figueroa PJ, Leite NJ, Barros RML. A flexible software for tracking of markers used in human motion analysis. Comput Methods Programs Biomed. 2003;72:155-65.

19. Andrade LA. Análise da marcha: protocolo experimental a partir de variáveis cinemáticas e antropométricas [dissertação]. Campinas: Faculdade de Educação Física, Universidade Estadual de Campinas; 2002. 
20. Pretchl HFR, Beintema DJ. The neurological examination of the fullterm newborn infant. Clin Dev Med. 1964:12:173.

21. Jeng SF, Chen LC, Yau KIT. Kinematic analysis of kicking movements in preterm infants with very low birth weight and full-term infants. Phys Ther. 2002;82(2):148-59.

22. Landgraf JF, Tudella E. Efeitos do peso externo nos chutes espontâneos de lactentes nos primeiros dois meses de vida. Rev Bras Fisioter. 2008;12:468-75
23. Thelen E, Fisher DM. The organization of spontaneous leg movements in newborn infants. J Motor Behav. 1983:15(4):353-77.

24. Piek JP. A longitudinal study of interlimb and intralimb coordination in full term and preterm infants. Inf Behav Dev. 1998;21:621.

25. Thelen E, Ridley-Johnson R, Fisher DM. Shifting patterns of bilateral coordination and lateral dominance in the leg movements of young infants. Dev Psychobiol. 1983;16(1):29-46. 